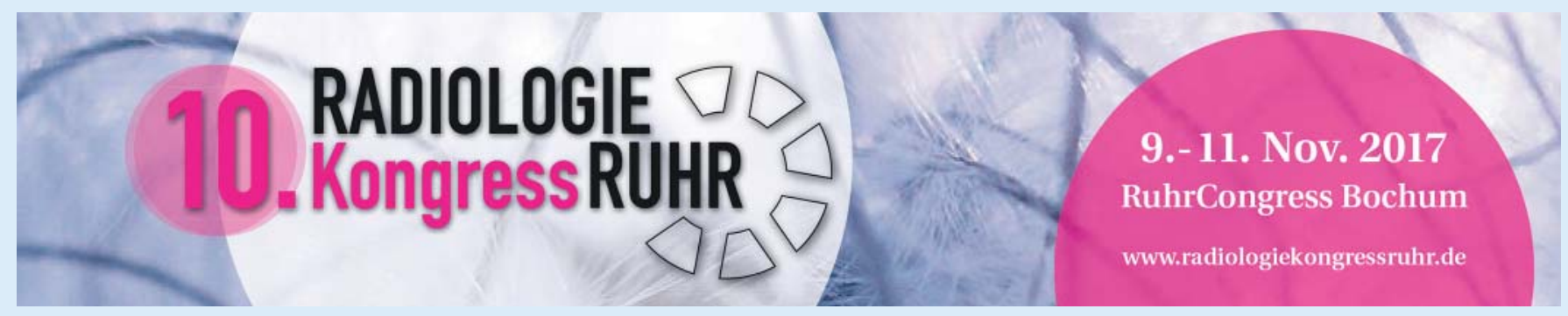

10. Radiologie Kongress Ruhr, 9.-11.11.2017 in Bochum

\title{
Hands-on-Workshops: Praktisch lernen auf dem 10. RKR
}

Der RadiologieKongressRuhr (RKR) ist seit jeher ein Kongress, der seinen Fokus auf die Fortbildung legt. Hier geht es um die Inhalte, Fertigkeiten und Techniken, die Radiologen in ihrer Praxis oder in ihrer Klinik Tag für Tag anwenden müssen. So hatten und haben die Vorträge in den Hauptsessions das Format von Refresherkursen und bauen mit dem TED-System eine sofortige Überprüfung des erlernten Wissens mit ein.

Seit einigen Jahren werden diese Vorträge nun auch durch Hands-on-Workshops ergänzt. Aus gleich drei verschiedenen Themenangeboten können die Teilnehmenden im Jubiläumsjahr 2017 wählen. Im Handson-Workshop „Stroke“ treten sechs Firmen auf, die Instrumente für die Thrombektomie herstellen. An Modellen werden diese zeigen, wie die verschiedenen Devices funktionieren. An zwei Simulatoren können die Teilnehmenden dann selbst Hand anlegen und sich ausprobieren. Im Hands-on-Workshop „periphere Interventionen“ präsentieren fünf Firmen ihre technischen Geräte für die interventionellen Eingriffe. Auch hier können die Teilnehmenden an Simulatoren ihre praktischen Fähigkeiten erweitern und vertiefen. Zum ersten Mal wird in diesem Jahr ein dritter Workshop zum Thema „perkutane Verfahren“ angeboten. Hier zeigen vier Firmen ihre technischen Lösungen und demonstrieren an Simulatoren die Herangehens- und Funktionsweise.

Für jede Arbeitsstation in allen Workshops wird ein erfahrener Arzt die Geräte erklären und gemeinsam mit den Firmenvertretern Fragen beantworten und diskutieren. Die Teilnehmerzahl ist mit zwei Personen pro Firma und Modell bewusst klein gehalten, damit eine intensive Lernatmosphäre herrscht und genügend Zeit zum praktischen Ausprobieren bleibt.
Im Folgenden finden Sie die Daten der Workshops in der Übersicht:

Donnerstag, 9. November 2017

14:00 - 17:00 Hands-on Workshop Stroke, Tagungsraum 2

Max. Teilnehmerzahl: 12 Personen pro Gruppe

Vorsitz: Prof. Dr. Bernd Turowski, Düsseldorf/Prof. Dr. Stefan Rohde, Dortmund

Es werden Lösungen der Firmen Stryker, Neuravi, Phenox, Penumbra, Acandis und Medtronic präsentiert.

Zielgruppe: Kollegen, die mit der Thrombektomie anfangen wollen oder noch am Anfang ihrer Erfahrung sind, können das Verfahren kennenlernen und sich von erfahrenen Kollegen Tipps zur Anwendung holen. Erfahrenere Kollegen können sich über die Unterschiede zwischen den verschiedenen Firmen informieren. Wer einfach mal sehen will, was Thrombektomie eigentlich heißt, ist auch willkommen.

14:00 - 15:00 Gruppe 1

Dr. Hannes Nordmeyer, Essen/Dr. Frauke Pult, Dortmund/Dr. Christoph Kabbasch, Köln/Dr. Stephan Haller, Bochum/Dr. Michael Oelerich, Hamm/ Bastian Kraus, Düsseldorf

15:00 - 16:00 Gruppe 2

Dr. Hannes Nordmeyer, Essen/Dr. Frauke Pult, Dortmund/Dr. Christoph Kabbasch, Köln/Dr. Stephan Haller, Bochum/Dr. Michael Oelerich, Hamm/ Bastian Kraus, Düsseldorf

16:00 - 17:00 Gruppe 3

Dr. Hannes Nordmeyer, Essen/Dr. Frauke Pult, Dortmund/Dr. Christoph Kabbasch, Köln/Dr. Stephan Haller, Bochum/Dr. Michael Oelerich, Hamm/ Bastian Kraus, Düsseldorf
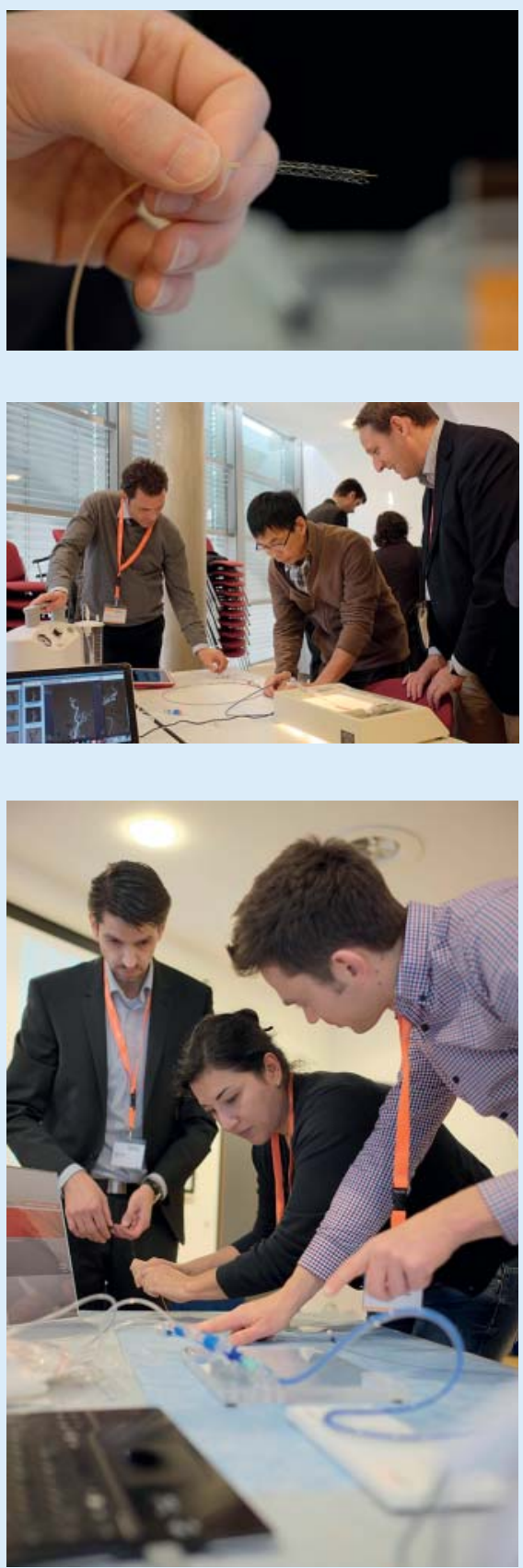

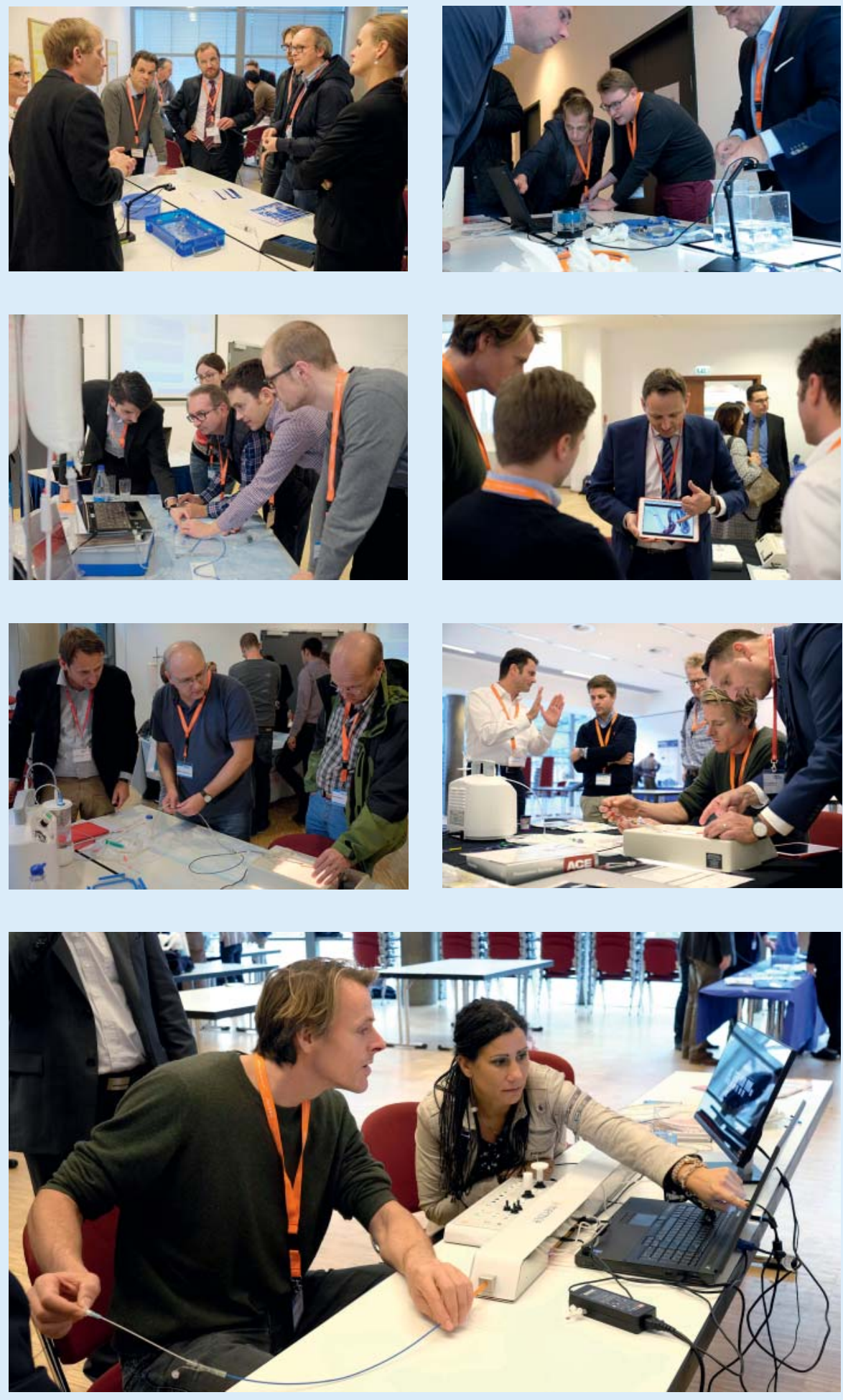

14:00 - 17:00 Hands-on Workshop periphere Interventionen, Tagungsraum 3

Max. Teilnehmerzahl: 10 Personen pro Gruppe

Vorsitz: Prof. Dr. Ralph, Kickuth, Würzburg/Prof. Dr. Patrick Haage, Wuppertal/Prof. Dr. Marcus Katoh, Krefeld 14:00 - 15:00 Gruppe 1

Anne-Marie Augustin, Würzburg/N.N. 15:00 - 16:00 Gruppe 2

Anne-Marie Augustin, Würzburg/N.N. 16:00 - 17:00 Gruppe 3

Anne-Marie Augustin, Würzburg/N.N.

14:00 - 17:00 Hands-on Workshop perkutane Verfahren, Künstlergarderobe 4

Max. Teilnehmerzahl: 8 Personen pro Gruppe

Vorsitz: Prof. Dr. Ralph Kickuth, Würzburg/Prof. Dr. Patrick Haage, Wuppertal/Prof. Dr. Marcus Katoh, Krefeld 14:00 - 15:00 Gruppe 1

Dr. Friederika Fluck, Würzburg/N.N. 15:00 - 16:00 Gruppe 2

Dr. Friederika Fluck, Würzburg/N.N. 16:00 - 17:00 Gruppe 3

Dr. Friederika Fluck, Würzburg/N.N.

Die Inhalte der Gruppen in einem Workshop sind identisch, Sie müssen sich demnach nur für eine Gruppe pro Workshop anmelden. Die Teilnahmegebühr beträgt 30,00 Euro.

Hier kommen Sie direkt zur Anmeldung zum 10. RKR.

Hier finden Sie das komplette Kongressprogramm des 10. RKR.

Bildergalerie (copyright: DRG/M. Grosler) 